PROCEEDINGS OF THE AMERICAN MATHEMATICAL SOCIETY

Volume 124, Number 10, October 1996

\title{
COMMUTING HOLOMORPHIC FUNCTIONS AND HYPERBOLIC AUTOMORPHISMS
}

\author{
CHIARA DE FABRITIIS
}

(Communicated by Eric Bedford)

\begin{abstract}
We give a complete classification of the holomorphic self-maps of the unit ball of $\mathbf{C}^{n}$ into itself which commute with a given hyperbolic automorphism.
\end{abstract}

\section{INTRODUCTION}

Let $\Delta$ be the unit disc of $\mathbf{C}$, and let $\gamma$ be a hyperbolic automorphism of $\Delta$. In 1941 M.H. Heins (see [6]) proved that, if a holomorphic map $f \in \operatorname{Hol}(\Delta, \Delta)$ from the unit disc $\Delta$ into itself commutes with $\gamma$ (under composition), then $f$ is either the identity map on $\Delta$, or it is a hyperbolic automorphism of $\Delta$ with the same fixed points of $\gamma$.

If one considers the unit ball $\Delta_{n}$ of $\mathbf{C}^{n}$ for $n>1$, then the study of the class of all holomorphic maps $f \in \operatorname{Hol}\left(\Delta_{n}, \Delta_{n}\right)$ which commute with a given hyperbolic automorphism $\gamma$ of $\Delta_{n}$ is still open. The author, together with Gentili (see [4]), contributed to this subject by obtaining information on the "structure" of the maps $f$ which commute with $\gamma$, under the hypothesis of "regularity" at one of the fixed points of $\gamma$ in $\partial \Delta_{n}$.

In this paper a complete classification of all the holomorphic maps of $\Delta_{n}$ into itself which commute with a given hyperbolic automorphism $\gamma$ of $\Delta_{n}$, for $n>1$, is obtained (Theorem 2.5). In dimension greater than one, the results turn out to be very different from those obtained by Heins for the unit disc of $\mathbf{C}$. A map $f \in \operatorname{Hol}\left(\Delta_{n}, \Delta_{n}\right)$ which commutes with a hyperbolic automorphism $\gamma$ need not be an automorphism of $\Delta_{n}$; instead a large class of non-automorphisms which commute with $\gamma$ is found and classified (Theorem 2.5 and Corollary 2.7).

In dimension 2 , the results obtained also provide information on the fixed points set of a map $f$ which commutes with $\gamma$; still the results differ much from those obtained in the one-dimensional case (Proposition 2.9).

Preliminaries and notation can be found in [10], [1] and [4].

\section{Preliminary Results}

In this section we recall some results which will be useful in the sequel. The proof of the following theorem can be found, e.g., in [1].

Received by the editors December 18, 1994.

1991 Mathematics Subject Classification. Primary 32A10; Secondary 30E25, 32A40, 32A30.

Key words and phrases. Commuting functions, hyperbolic automorphism, Wolff point.

(c)1996 American Mathematical Society 
Theorem 1.1. Each element $\gamma$ of the group Aut $\Delta_{n}$ can be extended holomorphically to an open neighborhood of $\bar{\Delta}_{n}$ and, if $\gamma \neq i d_{\Delta_{n}}$, then either $\gamma$ has at least one fixed point in $\Delta_{n}$, or it has no fixed points in $\Delta_{n}$ and it has one or two fixed points in $\partial \Delta_{n}$.

The following definition is also classical.

Definition 1.1. If $\gamma$ has some fixed points in $\Delta_{n}$, then it is called elliptic; if $\gamma$ has no fixed points in $\Delta_{n}$ and one fixed point in $\partial \Delta_{n}$, then it is called parabolic; if $\gamma$ has no fixed points in $\Delta_{n}$ and two fixed points in $\partial \Delta_{n}$, then it is called hyperbolic.

In 1941 M.H. Heins proved the following

Theorem 1.2. Let $\gamma$ be a hyperbolic automorphism of $\Delta$, and let $f \in \operatorname{Hol}(\Delta, \Delta)$ be such that $f \circ \gamma=\gamma \circ f$. Then either $f=i d_{\Delta}$ or $f$ is a hyperbolic automorphism of $\Delta$ with the same fixed points of $\gamma$.

A proof of the above theorem can be found in [6] (for a more recent exposition of this and related results, see [1]): the proof relies upon the existence of the derivative of $f$ at the Wolff point.

From now on $\gamma$ will be a hyperbolic element of $A u t \Delta_{n}$. Since Aut $\Delta_{n}$ acts doubly transitively on $\partial \Delta_{n}$, we can suppose, up to conjugation in Aut $\Delta_{n}$, that the fixed points of $\gamma$ in $\partial \Delta_{n}$ are $e_{1}$ and $-e_{1}$, where $e_{j}$ denotes the $j$-th element of the standard basis of $\mathbf{C}^{n}$. Such a hyperbolic automorphism $\gamma$ of $\Delta_{n}$ can be expressed, up to conjugation in $A u t \Delta_{n}$, by

$$
\gamma(z)=\frac{\left(\cosh t_{0} z_{1}+\sinh t_{0}, e^{i \theta_{2}} z_{2}, \ldots, e^{i \theta_{n}} z_{n}\right)}{\sinh t_{0} z_{1}+\cosh t_{0}},
$$

where $t_{0} \in \mathbf{R}-\{0\}$ and $\theta_{2}, \ldots, \theta_{n} \in \mathbf{R}$. Therefore, if $\gamma$ is a hyperbolic automorphism of $\Delta_{n}$ and if $f \in \operatorname{Hol}\left(\Delta_{n}, \Delta_{n}\right)$, then in the search for the solutions of equation $f \circ \gamma=\gamma \circ f$, we can suppose that $\gamma$ is given by (1.1).

The following result is due to de Fabritiis and Gentili (see [4]).

Proposition 1.3. Let $\gamma \in$ Aut $\Delta_{n}$ be a hyperbolic automorphism as in (1.1), and let $f=\left(f_{1}, \ldots, f_{n}\right) \in \operatorname{Hol}\left(\Delta_{n}, \Delta_{n}\right)$. If $f \circ \gamma=\gamma \circ f$, then there exists $t_{1} \in \mathbf{R}$ such that

$$
f_{1}\left(z_{1}, 0, \ldots, 0\right)=\frac{\cosh t_{1} z_{1}+\sinh t_{1}}{\sinh t_{1} z_{1}+\cosh t_{1}} .
$$

The next result also appears in [4] and completely determines the behaviour of $f$ on the disc $\Delta \times\{0\}$.

Proposition 1.4. Let $\gamma \in$ Aut $\Delta_{n}$ be a hyperbolic automorphism as in (1.1), and let $f=\left(f_{1}, \ldots, f_{n}\right) \in \operatorname{Hol}\left(\Delta_{n}, \Delta_{n}\right)$ be such that $f \circ \gamma=\gamma \circ f$. Then $f_{2}\left(z_{1}, 0, \ldots, 0\right)=$ $\cdots=f_{n}\left(z_{1}, 0, \ldots, 0\right)=0$ for all $z_{1} \in \Delta$.

Now, if $f \in \operatorname{Hol}\left(\Delta_{n}, \Delta_{n}\right)$ is a map which commutes with the holomorphic automorphism $\gamma$ defined by (1.1), we want to study the behaviour of $f$ outside the disk $\Delta \times\{0\}$. At first we "transfer" the problem to the Siegel upper half-space $H_{n}=\left\{w \in \mathbf{C}^{n}: \operatorname{Im} w_{1}>\left|w_{2}\right|^{2}+\cdots+\left|w_{n}\right|^{2}\right\}$ via the Cayley transform $\mathcal{C}$ from $\Delta_{n}$ to $H_{n}$ given by

$$
\mathcal{C}(z)=\left(i \frac{1+z_{1}}{1-z_{1}}, \frac{i z_{2}}{1-z_{1}}, \ldots, \frac{i z_{n}}{1-z_{1}}\right)
$$


If $F=\mathcal{C} \circ f \circ \mathcal{C}^{-1}$ and $\mu=\mathcal{C} \circ \gamma \circ \mathcal{C}^{-1}$, then $\mu \in A u t H_{n}$ and the fact that $f$ and $\gamma$ commute is equivalent to the fact that $F$ and $\mu$ commute.

An expression for $\mu$ is easily recovered from the form of $\gamma$ (see (1.1)); it turns out that

$$
\mu(w)=\left(\lambda^{2} w_{1}, e^{i \theta_{2}} \lambda w_{2}, \ldots, e^{i \theta_{n}} \lambda w_{n}\right),
$$

where $\lambda=e^{t_{0}}$ (hence, by our assumptions, $\lambda \neq 1$ ). By Proposition 1.4 and by the definition of the Cayley transform we obtain:

Corollary 1.5. Let $\mu$ be a hyperbolic automorphism of $H_{n}$ given by (1.3), and let $F: H_{n} \rightarrow H_{n}$ be holomorphic and such that $F \circ \mu=\mu \circ F$. Then there exists $k>0$ such that

$$
F_{1}\left(w_{1}, 0, \ldots, 0\right)=k^{2} w_{1}
$$

and that

$$
F_{2}\left(w_{1}, 0, \ldots, 0\right)=\cdots=F_{n}\left(w_{1}, 0, \ldots, 0\right)=0
$$

\section{MAIN RESULTS}

In this section we study the family of all holomorphic self-maps of $\Delta_{n}$ which commute with a given hyperbolic automorphism of $\Delta_{n}(n \geq 1)$ without any condition on the "regularity" of the self-maps. As we have seen, via the Cayley transform, this is equivalent to studying the family of all holomorphic self-maps $F$ of the Siegel upper half-space $H_{n}$ which commute with a hyperbolic automorphism $\mu$ of $H_{n}$ given by (1.3).

We know that there exists $k \in \mathbf{R}^{+}$such that $F_{1}\left(w_{1}, 0, \ldots, 0\right)=k^{2} w_{1}$ and $F_{j}\left(w_{1}, 0, \ldots, 0\right)=0$ for all $j \geq 2$ and all $w_{1} \in H_{1}$ (see Corollary 1.5). Now equation (1.5) has very strong consequences on the form of $F_{2}$ if $n=2$ : since $F_{2}\left(w_{1}, 0\right)=0$, for all $w_{1} \in H_{1}$, then we can find a function $h$, holomorphic on $H_{2}$, such that $F_{2}(w)=w_{2} h(w)$. The fact that $F$ and $\mu$ commute yields that $e^{i \theta_{2}} w_{2} h(\mu(w))=e^{i \theta_{2}} w_{2} h(w)$ for all $w \in H_{2}$. Therefore

$$
h(\mu(w))=h(w) \quad \forall w \in H_{2} .
$$

In fact, if $w_{2} \neq 0$, equation (2.1) is obviously satisfied and, by continuity, it holds for all $w \in H_{2}$.

Equation (2.1) suggests the investigation of the action of the subgroup generated by $\mu$ on $H_{2}$, or on $H_{n}$ for $n \geq 1$. Let $\Gamma=\left\{\mu^{m}, m \in \mathbf{Z}\right\}$ be the subgroup of $A u t H_{n}$ generated by $\mu$.

Proposition 2.1. $\Gamma$ acts freely and properly discontinuously on $H_{n}$.

Proof. The fact that $\lambda \neq 1$ in (1.3) implies that $\Gamma$ acts freely on $H_{n}$. To prove that $\Gamma$ acts properly discontinuously, we can consider the case in which $\lambda>1$ (otherwise we consider $\mu^{-1}$ instead of $\mu$ ). Let $\tilde{w} \in H_{n}$ and set

$$
U(\tilde{w})=\overline{B(\tilde{w}, \rho)} \cap\left\{w \in \mathbf{C}^{n}:\left|\operatorname{Im} \tilde{w}_{1}-\operatorname{Im} w_{1}\right| \leq \frac{1}{4}\left(1-\frac{1}{\lambda^{2}}\right) \operatorname{Im} \tilde{w}_{1}\right\},
$$

where $B(\tilde{w}, \rho)$ is the ball of center $\tilde{w}$ and euclidean radius $\rho$ in $\mathbf{C}^{2}$. If $\rho \ll 1$, then $U(\tilde{w})$ is a compact neighborhood of $\tilde{w}$ contained in $H_{n}$. 
Now we prove that, if there exists $s \in \mathbf{Z}$ such that $\mu^{s}(U(\tilde{w})) \cap U(\tilde{w}) \neq \emptyset$, then $s=0$. Let $w \in U(\tilde{w})$ be such that $\mu^{s}(w) \in U(\tilde{w})$. Since $w \in U(\tilde{w})$, we have

$$
\operatorname{Im} w_{1} \geq\left(1-\frac{1}{4}\left(1-\frac{1}{\lambda^{2}}\right)\right) \operatorname{Im} \tilde{w}_{1} \geq \frac{3}{4} \operatorname{Im} \tilde{w}_{1} .
$$

The fact that $\mu^{s}(w)$ belongs to $U(\tilde{w})$ implies now that

$$
\left|\operatorname{Im} \tilde{w}_{1}-\lambda^{2 s} \operatorname{Im} w_{1}\right| \leq \frac{1}{4}\left(1-\frac{1}{\lambda^{2}}\right) \operatorname{Im} \tilde{w}_{1}
$$

and hence

$$
\begin{aligned}
\left|\lambda^{2 s}-1\right| \operatorname{Im} w_{1} & =\left|\operatorname{Im} w_{1}-\lambda^{2 s} \operatorname{Im} w_{1}\right| \\
& \leq\left|\operatorname{Im} w_{1}-\operatorname{Im} \tilde{w}_{1}\right|+\left|\lambda^{2 s} \operatorname{Im} w_{1}-\operatorname{Im} \tilde{w}_{1}\right| \leq \frac{1}{2}\left(1-\frac{1}{\lambda^{2}}\right) \operatorname{Im} \tilde{w}_{1} .
\end{aligned}
$$

This in turn implies that

$$
\operatorname{Im} w_{1} \leq \frac{1}{2}\left(1-\frac{1}{\lambda^{2}}\right) \operatorname{Im} \tilde{w}_{1}\left|\lambda^{2 s}-1\right|^{-1} .
$$

If $s>0$, since $\lambda>1$, then $\lambda^{2 s}-1 \geq \lambda^{2}-1$, and therefore we obtain that

$$
\operatorname{Im} w_{1} \leq \frac{1}{2}\left(1-\frac{1}{\lambda^{2}}\right) \operatorname{Im} \tilde{w}_{1}\left(\lambda^{2}-1\right)^{-1}=\frac{1}{2 \lambda^{2}} \operatorname{Im} \tilde{w}_{1}<\frac{3}{4} \operatorname{Im} \tilde{w}_{1},
$$

which contradicts $(2.2)$.

If $s<0$, then $\left|\lambda^{2 s}-1\right|=1-\lambda^{2 s} \geq 1-\lambda^{-2}$, therefore we have

$$
\operatorname{Im} w_{1} \leq \frac{1}{2}\left(1-\frac{1}{\lambda^{2}}\right) \operatorname{Im} \tilde{w}_{1}\left(1-\frac{1}{\lambda^{2}}\right)^{-1}=\frac{1}{2} \operatorname{Im} \tilde{w}_{1}<\frac{3}{4} \operatorname{Im} \tilde{w}_{1},
$$

which again contradicts (2.2). In conclusion $s=0$ and therefore $\Gamma$ acts properly discontinuously on $H_{n}$.

Let $\mu$ be a hyperbolic automorphism of $H_{n}$ as in (1.3), and let $F \in \operatorname{Hol}\left(H_{n}, H_{n}\right)$ be such that $F \circ \mu=\mu \circ F$. We will now prove a result on the structure of the last $n-1$ components of $F$.

Let $j$ be a natural number such that $1 \leq j \leq n$, and define $\Gamma_{j}$ to be the subgroup of $A u t H_{j}$ generated by the holomorphic automorphism of $H_{j}$ given by $\left(w_{1}, \ldots, w_{j}\right) \mapsto\left(\lambda^{2} w_{1}, \lambda e^{i \theta_{2}} w_{2}, \ldots, \lambda e^{i \theta_{j}} w_{j}\right)$, i.e. by the "restriction" of $\mu$ to $H_{j}$. Then Proposition 2.1 implies that $\Gamma_{j}$ acts freely and properly discontinuously on $H_{j}$ for all $j \leq n$, and therefore we can endow $X_{j}=H_{j} / \Gamma_{j}$ with a complex structure such that the projection $\pi_{j}$ from $H_{j}$ to $X_{j}$ is holomorphic.

We will find a suitable form of the $m$-th component $F_{m}$ of $F$, for $2 \leq m \leq n$. Let $\ln : H_{1} \rightarrow \mathbf{C}$ be a branch of the logarithm on the upper half-plane in $\mathbf{C}$ and recall that, by Corollary $1.5, F_{m}\left(w_{1}, 0, \ldots, 0\right)=0$ for all $2 \leq m \leq n$.

Proposition 2.2. Let $F: H_{n} \rightarrow H_{n}$ be a holomorphic map which commutes with the hyperbolic automorphism $\mu$ given by (1.3). Then there exist $(n-1)^{2}$ holomorphic functions $\hat{g}_{j m}: X_{j} \rightarrow \mathbf{C}$ such that the $m$-th component $F_{m}$ of $F$ is given by

$$
F_{m}(w)=k \sum_{j=2}^{n} w_{j} e^{i\left(\theta_{m}-\theta_{j}\right) \log w_{1} / 2 \log \lambda} \hat{g}_{j m}\left(\pi_{j}\left(w_{1}, \ldots, w_{j}\right)\right) .
$$

Proof. First of all we prove that $F_{m}(w)=k \sum_{j=2}^{n} w_{j} g_{j m}\left(w_{1}, \ldots, w_{j}\right)$, where $g_{j m}$ are suitable holomorphic functions on $H_{j}$. 
Since $F_{m}\left(w_{1}, 0, \ldots, 0\right)=0$, the map $w_{2} \mapsto F_{m}\left(w_{1}, w_{2}, 0, \ldots, 0\right)$ is equal to 0 if $w_{2}=0$, and hence there exists a holomorphic map on $H_{2}$, say $g_{2 m}$, such that

$$
F_{m}\left(w_{1}, w_{2}, 0, \ldots, 0\right)=k w_{2} g_{2 m}\left(w_{1}, w_{2}\right) .
$$

Now the map $w_{3} \mapsto F_{m}\left(w_{1}, w_{2}, w_{3}, 0, \ldots, 0\right)-k w_{2} g_{2 m}\left(w_{1}, w_{2}\right)$ is equal to 0 if $w_{3}=0$, and hence there exists a holomorphic map on $H_{3}$, say $g_{3 m}$, such that

$$
F_{m}\left(w_{1}, w_{2}, w_{3}, 0, \ldots, 0\right)-k w_{2} g_{2 m}\left(w_{1}, w_{2}\right)=k w_{3} g_{3 m}\left(w_{1}, w_{2}, w_{3}\right) .
$$

By a recursive procedure, we obtain the existence of the functions $g_{j m}$ such that

$$
F_{m}(w)=k \sum_{j=2}^{n} w_{j} g_{j m}\left(w_{1}, \ldots, w_{j}\right) .
$$

Now we prove that such $g_{j m}$ 's are unique. In fact, if there are two families of holomorphic functions, say $g_{j m}$ and $p_{j m}$, from $H_{j}$ to $\mathbf{C}$ such that

$$
F_{m}(w)=k \sum_{j=2}^{n} w_{j} g_{j m}\left(w_{1}, \ldots, w_{j}\right)=k \sum_{j=2}^{n} w_{j} p_{j m}\left(w_{1}, \ldots, w_{j}\right),
$$

then the difference $g_{j m}-p_{j m}$ satisfies the equation

$$
\sum_{j=2}^{n} w_{j}\left(g_{j m}-p_{j m}\right)\left(w_{1}, \ldots, w_{j}\right)=0 \text { for all } w \in H_{n} .
$$

Let $w_{3}=\cdots=w_{n}=0$. Then $g_{2 m}\left(w_{1}, w_{2}\right)=p_{2 m}\left(w_{1}, w_{2}\right)$ for all $\left(w_{1}, w_{2}\right) \in H_{2}($ if $w_{2} \neq 0$ the assertion is obvious, otherwise we use a continuity argument). We proceed in the same way, taking $w_{4}=\cdots=w_{n}=0$ and we obtain $g_{3 m}\left(w_{1}, w_{2}, w_{3}\right)=$ $p_{3 m}\left(w_{1}, w_{2}, w_{3}\right)$ for all $\left(w_{1}, w_{2}, w_{3}\right) \in H_{3}$. In conclusion we obtain the uniqueness of the functions $g_{j m}$ recursively.

Now we will get information about the behaviour of the functions $g_{j m}$. In order to simplify notation we will write $\mu\left(w_{1}, \ldots, w_{j}\right)$ to denote $\left(\lambda^{2} w_{1}, \lambda e^{i \theta_{2}} w_{2}, \ldots, \lambda e^{i \theta_{j}} w_{j}\right)$. The fact that $F \circ \mu=\mu \circ F$ implies that (for any $2 \leq m \leq n$ )

$$
\sum_{j=2}^{n} w_{j}\left(e^{i \theta_{m}} g_{j m}\left(w_{1}, \ldots, w_{j}\right)-e^{i \theta_{j}} g_{j m}\left(\mu\left(w_{1}, \ldots, w_{j}\right)\right)\right)=0 .
$$

Then, for any $2 \leq j, m \leq n$,

$$
g_{j m}\left(w_{1}, \ldots, w_{j}\right)=e^{i\left(\theta_{j}-\theta_{m}\right)} g_{j m}\left(\mu\left(w_{1}, \ldots, w_{j}\right)\right)
$$

(for all $\left.\left(w_{1}, \ldots, w_{j}\right) \in H_{j}\right)$. Having defined

$$
\tilde{g}_{j m}\left(w_{1}, \ldots, w_{j}\right)=e^{i\left(\theta_{j}-\theta_{m}\right) \log w_{1} / 2 \log \lambda} g_{j m}\left(w_{1}, \ldots, w_{j}\right),
$$

it is easy to see that $\tilde{g}_{j m}$ is automorphic under the action of the "restriction" of $\mu$ to $H_{j}$. Therefore there exist holomorphic functions $\hat{g}_{j m}: X_{j} \rightarrow \mathbf{C}$ such that $\tilde{g}_{j m}\left(w_{1}, \ldots, w_{j}\right)=\hat{g}_{j m}\left(\pi_{j}\left(w_{1}, \ldots, w_{j}\right)\right)$, and this concludes the proof.

We turn our attention now to the investigation of the behaviour of the first component of $F$. We already proved that there exists a positive $k$ such that $F_{1}\left(w_{1}, 0, \ldots, 0\right)=k^{2} w_{1}$. Then we can write

$$
F_{1}(w)=k^{2}\left(w_{1}+\sum_{j=2}^{n} w_{j} \alpha_{j}\left(w_{1}\right)+P(w)\right)
$$


where $P\left(w_{1}, 0, \ldots, 0\right)=0$ and $\frac{\partial P}{\partial w_{j}}\left(w_{1}, 0, \ldots, 0\right)=0$ for all $w_{1} \in H_{1}$ and for $j=2, \ldots, n$. First of all we want to prove that the $\alpha_{j}$ 's vanish identically.

Proposition 2.3. Let $F$ be a holomorphic map from $H_{n}$ into itself which commutes with $\mu$ given by (1.3). Write $F_{1}$ as in (2.3). Then $\alpha_{j} \equiv 0$ for $j=2, \ldots, n$.

Proof. Since $\operatorname{Im} F_{1}(w)>\left|F_{2}(w)\right|^{2}+\cdots+\left|F_{n}(w)\right|^{2}$ for all $w \in H_{n}$, it follows that $\operatorname{Im} F_{1}(w)>0$ for all $w \in H_{n}$. Take $j \in\{2, \ldots, n\}$ and consider $F_{1}$ on $\left(w_{1}, 0, \ldots, 0, w_{j}, 0, \ldots, 0\right)$. Writing $w_{2}$ instead of $w_{j}$, it is enough to prove that $\alpha_{j}=0$ when $j=2$. Therefore it is enough to prove the statement when $n=2$. From now on we will denote $\alpha_{2}$ by $\alpha$. The fact that $P\left(w_{1}, 0\right)=0$ and $\frac{\partial P}{\partial w_{2}}\left(w_{1}, 0\right)=0$ for all $w_{1} \in H_{1}$ implies that there exists a function $\eta$, holomorphic on $H_{2}$, such that $P\left(w_{1}, w_{2}\right)=w_{2}^{2} \eta\left(w_{1}, w_{2}\right)$. Since $\operatorname{Im} F_{1}\left(w_{1}, w_{2}\right)>0$ for all $\left(w_{1}, w_{2}\right) \in H_{2}$, we have

$$
\operatorname{Im} w_{1}>-\operatorname{Im}\left(\alpha\left(w_{1}\right) w_{2}+\eta(w) w_{2}^{2}\right)
$$

for all $\left(w_{1}, w_{2}\right) \in H_{2}$. Take $w_{1}^{0} \in \mathbf{C}$ such that $\operatorname{Im} w_{1}^{0}>0$. Then, for any $w_{2} \in \mathbf{C}$ such that $\left|w_{2}\right|^{2}<\operatorname{Im} w_{1}^{0}$, the point $\left(w_{1}^{0}, w_{2}\right)$ belongs to $H_{2}$. Let $\varepsilon \in \mathbf{R}$ be such that $0<\varepsilon<\operatorname{Im} w_{1}^{0}$ and set $R=\sqrt{\operatorname{Im} w_{1}^{0}-\varepsilon}, \quad r=R / 2$. The Borel-Carathéodory theorem and inequality (2.4) imply now that

$$
\begin{aligned}
& \max _{w_{2} \in \Delta_{r}}\left|\alpha\left(w_{1}^{0}\right) w_{2}+w_{2}^{2} \eta\left(w_{1}^{0}, w_{2}\right)\right| \\
& \quad \leq \frac{2 r}{R-r} \max _{w_{2} \in \partial \Delta_{r}} \operatorname{Im}\left(-\left(\alpha\left(w_{1}^{0}\right) w_{2}+\eta\left(w_{1}^{0}, w_{2}\right) w_{2}^{2}\right)\right) \\
& \quad \leq \frac{2 r}{R-r} \max _{w_{2} \in \partial \Delta_{r}} \operatorname{Im} w_{1}^{0}=2 \operatorname{Im} w_{1}^{0} .
\end{aligned}
$$

We pass to the evaluation of the maximum modulus of the function $w_{2} \mapsto$ $w_{2}^{2} \eta\left(w_{1}^{0}, w_{2}\right)+w_{2} \alpha\left(w_{1}^{0}\right)$ on $\Delta_{r}$, and obtain

$$
\begin{aligned}
2 \operatorname{Im} w_{1}^{0} & \geq \max _{w_{2} \in \Delta_{r}}\left|w_{2}^{2} \eta\left(w_{1}^{0}, w_{2}\right)+w_{2} \alpha\left(w_{1}^{0}\right)\right|=\max _{w_{2} \in \partial \Delta_{r}}\left|w_{2}^{2} \eta\left(w_{1}^{0}, w_{2}\right)+w_{2} \alpha\left(w_{1}^{0}\right)\right| \\
& =\frac{1}{2} \sqrt{\operatorname{Im} w_{1}^{0}-\varepsilon} \max _{w_{2} \in \partial \Delta_{r}}\left|w_{2} \eta\left(w_{1}^{0}, w_{2}\right)+\alpha\left(w_{1}^{0}\right)\right| \\
& =\frac{1}{2} \sqrt{\operatorname{Im} w_{1}^{0}-\varepsilon} \max _{w_{2} \in \Delta_{r}}\left|w_{2} \eta\left(w_{1}^{0}, w_{2}\right)+\alpha\left(w_{1}^{0}\right)\right| .
\end{aligned}
$$

Taking the limit for $\varepsilon \rightarrow 0^{+}$we get

$$
\left|w_{2} \eta\left(w_{1}^{0}, w_{2}\right)+\alpha\left(w_{1}^{0}\right)\right| \leq 4\left(\operatorname{Im} w_{1}^{0}\right)^{1 / 2}
$$

for all $w_{2} \in \mathbf{C}$ such that $\left|w_{2}\right|^{2}<\operatorname{Im} w_{1}^{0}$. The number $\alpha\left(w_{1}^{0}\right)$ is the value at 0 of the holomorphic function $w_{2} \mapsto w_{2} \eta\left(w_{1}^{0}, w_{2}\right)+\alpha\left(w_{1}^{0}\right)$ and therefore inequality (2.6) implies that

$$
\left|\alpha\left(w_{1}^{0}\right)\right| \leq 4\left(\operatorname{Im} w_{1}^{0}\right)^{1 / 2}
$$

for all $w_{1}^{0} \in \mathbf{C}$ such that $\operatorname{Im} w_{1}^{0}>0$.

Inequality (2.7) implies now that, for any $\tau \in \mathbf{R}$,

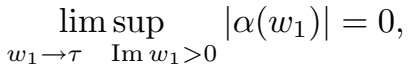

and the reflection principle yields that there exists an entire function $\tilde{\alpha}$ which extends $\alpha$. Inequality (2.7) entails that $\tilde{\alpha}(w)=0$ for all $w \in \mathbf{R}$. Therefore $\tilde{\alpha} \equiv 0$ and hence $\alpha \equiv 0$, which proves the proposition. 
Thus far we have proved that the function $F_{1}$ has the form

$$
F_{1}(w)=k^{2}\left(w_{1}+P(w)\right)
$$

where $P\left(w_{1}, 0, \ldots, 0\right)=0$ and $\frac{\partial P}{\partial w_{j}}\left(w_{1}, 0, \ldots, 0\right)=0$ for all $w_{1} \in H_{1}$ and for $j=2, \ldots, n$. Now we want to give a "standard" form to the function $P$ and use this form to give a complete classification of the holomorphic self-maps of $H_{n}$ which commute with $\mu$ given by (1.3).

Proposition 2.4. Let $P$ be a holomorphic function on $H_{n}$ such that $P\left(w_{1}, 0, \ldots, 0\right)$ $=0$ and $\frac{\partial P}{\partial w_{j}}\left(w_{1}, 0, \ldots, 0\right)=0$ for all $w_{1} \in H_{1}$ and for $j=2, \ldots, n$. Then there exist $n(n-1) / 2$ holomorphic functions $\beta_{j l}: H_{j} \rightarrow \mathbf{C}$ for $2 \leq j \leq l \leq n$ such that $P(w)=\sum_{2 \leq j \leq l \leq n} w_{j} w_{l} \beta_{j l}\left(w_{1}, \ldots, w_{j}\right)$. Moreover the family $\left\{\beta_{j l}\right\}(2 \leq j \leq l \leq n)$ is unique.

Proof. First of all we prove the assertion on uniqueness: if there are two families of functions with the required properties, then there exists a family $\left\{d_{j l}\right\}$ such that

$$
\sum_{2 \leq j \leq l \leq n} w_{j} w_{l} d_{j l}\left(w_{1}, \ldots, w_{j}\right)=0
$$

on $H_{n}$. Let $w_{3}=\cdots=w_{n}=0$. Then $w_{2}^{2} d_{22}\left(w_{1}, w_{2}\right)=0$ for all $\left(w_{1}, w_{2}\right) \in H_{2}$ and therefore $d_{22} \equiv 0$. Let $w_{3}=\cdots \widehat{w}_{j} \cdots=w_{n}=0$; then $w_{2} w_{j} d_{2 j}\left(w_{1}, w_{2}\right)+$ $w_{j}^{2} d_{j j}\left(w_{1}, w_{2}, 0, w_{j}, 0,0\right)=0$ and therefore $d_{2 j} \equiv 0$ on $H_{2}$. By iterating the above procedure, we obtain the uniqueness of the family $\left\{\beta_{j l}\right\}$.

We pass now to the proof of the existence of such a family of functions $\left\{\beta_{j l}\right\}$ as in the statement.

Since $P\left(w_{1}, 0, \ldots, 0\right)=0$ and $\frac{\partial P}{\partial w_{j}}\left(w_{1}, 0, \ldots, 0\right)=0$ (for $j=2, \ldots, n$ and for all $\left.w_{1} \in H_{1}\right)$, then there exist $\beta_{2 j}: H_{2} \rightarrow \mathbf{C}$, holomorphic, such that $w_{2}^{2} \beta_{22}\left(w_{1}, w_{2}\right)=$ $P\left(w_{1}, w_{2}, 0, \ldots, 0\right)$ and that $w_{2} \beta_{2 j}\left(w_{1}, w_{2}\right)=\frac{\partial P}{\partial w_{j}}\left(w_{1}, w_{2}, 0, \ldots, 0\right)$ (for $j=3, \ldots, n$ and for all $\left.\left(w_{1}, w_{2}\right) \in H_{2}\right)$.

Set $P_{1}(w)=P(w)-w_{2}^{2} \beta_{22}\left(w_{1}, w_{2}\right)-w_{2} w_{3} \beta_{23}\left(w_{1}, w_{2}\right)-\cdots-w_{2} w_{n} \beta_{2 n}\left(w_{1}, w_{2}\right)$. It is easy to see that $P_{1}\left(w_{1}, w_{2}, 0, \ldots, 0\right)=0$ and $\frac{\partial P_{1}}{\partial w_{j}}\left(w_{1}, w_{2}, 0, \ldots, 0\right)=0$ for $j=3, \ldots, n$ and for all $\left(w_{1}, w_{2}\right) \in H_{2}$. Then there exist $\beta_{3 j}: H_{3} \rightarrow \mathbf{C}$, holomorphic, such that $w_{3}^{2} \beta_{33}\left(w_{1}, w_{2}, w_{3}\right)=P_{1}\left(w_{1}, w_{2}, w_{3}, 0, \ldots, 0\right)$ and $w_{3} \beta_{3 j}\left(w_{1}, w_{2}, w_{3}\right)=$ $\frac{\partial P_{1}}{\partial w_{j}}\left(w_{1}, w_{2}, w_{3}, 0, \ldots, 0\right)$ for $j=4, \ldots, n$ and for all $\left(w_{1}, w_{2}, w_{3}\right) \in H_{3}$. By iterating this procedure, we end with a holomorphic function $\beta_{n n}: H_{n} \rightarrow \mathbf{C}$ such that $P_{n-2}(w)=w_{n}^{2} \beta_{n n}(w)$. This proves the existence of the required family.

By gathering together all the results, we have proved that, if $F: H_{n} \rightarrow H_{n}$ is a holomorphic map which commutes with $\mu$ given by (1.3), then there exist: $k>0$, $\beta_{j l} \in \operatorname{Hol}\left(H_{j}, \mathbf{C}\right)(2 \leq j \leq l \leq n)$ and $\hat{g}_{j m} \in \operatorname{Hol}\left(X_{j}, \mathbf{C}\right)(j, m=2, \ldots, n)$ such that

$$
F_{1}(w)=k^{2}\left(w_{1}+\sum_{2 \leq j \leq l \leq n} w_{j} w_{l} \beta_{j l}\left(w_{1}, \ldots, w_{j}\right)\right)
$$


and

$$
F_{m}(w)=k \sum_{j=2}^{n} w_{j} e^{i\left(\theta j-\theta_{m}\right) \log w_{1} / 2 \log \lambda} \hat{g}_{j m}\left(\pi_{j}\left(w_{1}, \ldots, w_{j}\right)\right) .
$$

The fact that $F$ commutes with $\mu$ and the uniqueness of the family $\beta_{j m}$ imply that

$$
e^{i\left(\theta_{j}+\theta_{l}\right)} \beta_{j l}(\mu(w))=\beta_{j l}(w) \quad \forall w \in H_{j} .
$$

Let us define

$$
\tilde{\beta}_{j l}(w)=e^{i\left(\theta_{j}+\theta_{l}\right) \log w_{1} / 2 \log \lambda} \beta_{j l}(w) ;
$$

then equation (2.8) entails that $\tilde{\beta}_{j l}$ is automorphic under the action of $\Gamma_{j}$. Therefore, if $F: H_{n} \rightarrow H_{n}$ is a holomorphic map which commutes with $\mu$ given by (1.3), we can find $k>0, \hat{g}_{j l}, \hat{\beta}_{j l} \in \operatorname{Hol}\left(X_{j}, \mathbf{C}\right)$ such that

$$
\begin{gathered}
F_{1}(w)=k^{2}\left(w_{1}+\sum_{2 \leq j \leq l \leq n} w_{j} w_{l} e^{-i\left(\theta_{j}+\theta_{l}\right) \log w_{1} / 2 \log \lambda} \hat{\beta}_{j l}\left(\pi_{j}\left(w_{1}, \ldots, w_{j}\right)\right)\right) \quad \text { and } \\
F_{m}(w)=k \sum_{j=2}^{n} w_{j} e^{i\left(\theta_{m}-\theta_{j}\right) \log w_{1} / 2 \log \lambda} \hat{g}_{j m}\left(\pi_{j}\left(w_{1}, \ldots, w_{j}\right)\right)
\end{gathered}
$$

for $m=2, \ldots, n$.

Moreover it is easy to see that, if $F$ has the above form, then $F: H_{n} \rightarrow \mathbf{C}^{n}$ commutes with $\mu$ given by (1.3) (to be more precise, with the holomorphic extension of $\mu$ to $\mathbf{C}^{n}$ ).

Then we need only a "restriction of the image" to obtain a complete classification of the holomorphic maps from $H_{n}$ into itself which commute with $\mu$. Now we can state:

Theorem 2.5. Let $F: H_{n} \rightarrow H_{n}$ be a holomorphic map which commutes with $\mu$, the hyperbolic automorphism of $H_{n}$ given by (1.3). Then there exist $k>0$, $\hat{g}_{j l} \in \operatorname{Hol}\left(X_{j}, \mathbf{C}\right)($ for $j, l=2, \ldots, n)$ and $\hat{\beta}_{j l} \in \operatorname{Hol}\left(X_{j}, \mathbf{C}\right)($ for $2 \leq j \leq l \leq n)$ such that

$$
\begin{gathered}
F_{1}(w)=k^{2}\left(w_{1}+\sum_{2 \leq j \leq l \leq n} w_{j} w_{l} e^{-i\left(\theta_{j}+\theta_{l}\right) \log w_{1} / 2 \log \lambda} \hat{\beta}_{j l}\left(\pi_{j}\left(w_{1}, \ldots, w_{j}\right)\right)\right), \\
F_{m}(w)=k \sum_{j=2}^{n} w_{j} e^{i\left(\theta_{m}-\theta_{j}\right) \log w_{1} / 2 \log \lambda} \hat{g}_{j m}\left(\pi_{j}\left(w_{1}, \ldots, w_{j}\right)\right)
\end{gathered}
$$

for $m=2, \ldots, n$ and that

$$
\begin{aligned}
& \operatorname{Im}\left(w_{1}+\sum_{2 \leq j \leq l \leq n} w_{j} w_{l} e^{-i\left(\theta_{j}+\theta_{l}\right) \log w_{1} / 2 \log \lambda} \hat{\beta}_{j l}\left(\pi_{j}\left(w_{1}, \ldots, w_{j}\right)\right)\right) \\
& \quad>\sum_{m=2}^{n}\left|\sum_{j=2}^{n} w_{j} e^{i\left(\theta_{m}-\theta_{j}\right) \log w_{1} / 2 \log \lambda} \hat{g}_{j m}\left(\pi_{j}\left(w_{1}, \ldots, w_{j}\right)\right)\right|^{2} \quad \forall w \in H_{n} .
\end{aligned}
$$

Vice versa, let $F$ be as in (2.9) and (2.10), where $\hat{g}_{j m}, \hat{\beta}_{j m} \in \operatorname{Hol}\left(X_{j}, \mathbf{C}\right)$ satisfy (2.11). Then $F: H_{n} \rightarrow H_{n}$ commutes with $\mu$. Moreover, the map which associates $F$ to $\left(k, \hat{g}_{j m}, \hat{\beta}_{j m}\right)$ is one-to-one. 
The above theorem gives a complete answer to the problem of finding all holomorphic self-maps of $H_{n}$ which commute with the hyperbolic automorphism $\mu$ given by (1.3). By conjugation - as remarked at the beginning of this paper - it also gives an answer to the problem of finding all holomorphic self-maps of $H_{n}$ which commute with a given hyperbolic automorphism of $H_{n}$. Notice that condition (2.11) is fulfilled if the modulus of the $\hat{g}_{j m}$ and $\hat{\beta}_{j m}$ is very small. Therefore an open neighborhood of 0 in $\mathrm{Hol}\left(X_{2}, \mathbf{C}\right) \times \cdots \times H o l\left(X_{n}, \mathbf{C}\right)$ satisfies condition (2.11). This shows how deep the difference between the one-dimensional case and the multidimensional case is (for the one-dimensional case see, e.g., [1] or [6]).

Now we will consider, with particular attention, the case $n=2$, in which some other results can be given. First of all we restate Theorem 2.5 for $n=2$.

Theorem 2.6. Let $\mathrm{F}: \mathrm{H}_{2} \rightarrow \mathrm{H}_{2}$ be a holomorphic map which commutes with the hyperbolic automorphism $\mu$ given by (1.3). Then there exist $k>0$ and $\hat{g}, \hat{\beta} \in$ $\mathrm{Hol}\left(\mathrm{X}_{2}, \mathbf{C}\right)$ such that

a) $F(w)=\left(k^{2}\left(w_{1}+w_{2}^{2} e^{-i \theta_{2} \log w_{1} / \log \lambda} \hat{\beta}(\pi(w))\right), k w_{2} \hat{g}(\pi(w))\right)$ and

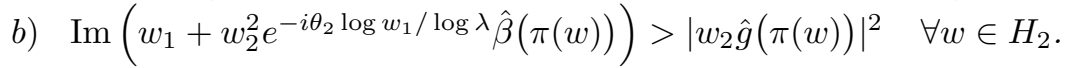

Vice versa, let $F$ be as in $a)$, where $\hat{g}, \hat{\beta} \in \operatorname{Hol}\left(X_{2}, \mathbf{C}\right)$ satisfy $\left.b\right)$. Then $F: H_{2} \rightarrow H_{2}$ commutes with $\mu$. Moreover, the map which associates $F$ to $(k, \hat{g}, \hat{\beta})$ is one-to-one.

Notice that condition $b)$ is satisfied if $e^{\theta_{2} \pi /|\log \lambda|}|\hat{\beta}(\pi(w))|+|\hat{g}(\pi(w))|^{2} \leq 1$. Therefore an open neighborhood of 0 in $\operatorname{Hol}\left(X_{2}, \mathbf{C}\right) \times \operatorname{Hol}\left(X_{2}, \mathbf{C}\right)$ consists of maps satisfying condition $b$ ).

There are "many" functions $f$ which commute with $\gamma$ : we will study in detail the "richness" of the class of functions given in Theorem 2.6, when $\hat{\beta}=0$.

Corollary 2.7. Let $\mathrm{F}: \mathrm{H}_{2} \rightarrow \mathrm{H}_{2}$ be a holomorphic map which commutes with the hyperbolic automorphism $\mu$ given by (1.3). If $F_{1}$ does not depend on $w_{2}$, then there exist $k>0$ and $\hat{g} \in \operatorname{Hol}\left(X_{2}, \mathbf{C}\right)$ such that

a) $F(w)=\left(k^{2} w_{1}, k w_{2} \hat{g}(\pi(w))\right)$ and

b) $|\hat{g}(\pi(w))| \leq 1 \quad \forall w \in H_{2}$.

Vice versa, let $F$ be as in $a)$, where $\hat{g} \in \operatorname{Hol}\left(X_{2}, \mathbf{C}\right)$ satisfies $\left.b\right)$. Then $F: H_{2} \rightarrow H_{2}$ commutes with $\mu$.

Proof. By Theorem 2.6, if $F$ is a holomorphic self-map of $H_{2}$ which commutes with $\mu$, then $F_{1}\left(w_{1}, 0\right)=k^{2} w_{1}$ for a suitable $k>0$.

The fact that $F_{1}$ does not depend on $w_{2}$ implies that

$$
F(w)=\left(k^{2} w_{1}, k w_{2} \hat{g}(\pi(w))\right)
$$

Moreover, that fact that $F$ maps $H_{2}$ into itself entails that

$$
\operatorname{Im} k^{2} w_{1}>k^{2}\left|w_{2}\right|^{2}|\hat{g}(\pi(w))|^{2} \quad \forall w \in H_{2}
$$


Consider $w_{1}^{0} \in \mathbf{C}$ such that $\operatorname{Im} w_{1}^{0}>0$, and let $\varepsilon \in \mathbf{R}$ be such that $0<\varepsilon<\operatorname{Im} w_{1}^{0}$. Define $r(\varepsilon)=\sqrt{\operatorname{Im} w_{1}^{0}-\varepsilon}$. Inequality (2.12) now implies that

$$
\begin{aligned}
\operatorname{Im} w_{1}^{0} & >\max _{w_{2} \in \Delta_{r(\varepsilon)}}\left|w_{2}\right|^{2}\left|\hat{g}\left(\pi\left(w_{1}^{0}, w_{2}\right)\right)\right|^{2}=\max _{w_{2} \in \partial \Delta_{r(\varepsilon)}}\left|w_{2}\right|^{2}\left|\hat{g}\left(\pi\left(w_{1}^{0}, w_{2}\right)\right)\right|^{2} \\
& =\left(\operatorname{Im} w_{1}^{0}-\varepsilon\right) \max _{w_{2} \in \partial \Delta_{r(\varepsilon)}}\left|\hat{g}\left(\pi\left(w_{1}^{0}, w_{2}\right)\right)\right|^{2} \\
& =\left(\operatorname{Im} w_{1}^{0}-\varepsilon\right) \max _{w_{2} \in \Delta_{r(\varepsilon)}}\left|\hat{g}\left(\pi\left(w_{1}^{0}, w_{2}\right)\right)\right|^{2} .
\end{aligned}
$$

Taking the limit for $\varepsilon \rightarrow 0^{+}$we obtain that $\left|\hat{g}\left(\pi\left(w_{1}^{0}, w_{2}\right)\right)\right|^{2} \leq 1$ for all $w_{2} \in \mathbf{C}$ such that $\left|w_{2}\right|^{2}<\operatorname{Im} w_{1}^{0}$. This in turn implies that, for any $w \in H_{2},|\hat{g}(\pi(w))| \leq 1$.

The sufficiency of $a$ ) and $b$ ) is obvious.

The above Corollary shows how large the family of all holomorphic self-maps of $\mathrm{H}_{2}$ which commute with a given hyperbolic automorphism of $\mathrm{H}_{2}$ is. In fact, if $\hat{g} \circ \pi$ does not depend on $w_{2}$, then $\hat{g} \circ \pi$ is a holomorphic map from the annulus $A(\rho, 1)=\{z \in \mathbf{C}: \rho<|z|<1\}$ (where $\rho=\exp \left(-\pi^{2} /|\log \lambda|\right)$ ) to the closed unit disk $\bar{\Delta}$. It is well known that this space of functions is quite large.

Theorem 2.6 also makes it possible to study the fixed points set of a holomorphic self-map of $\Delta_{2}$ which commutes with a given hyperbolic automorphism $\gamma$. As we are interested in the structure of $f i x f=\left\{z \in \Delta_{2}: f(z)=z\right\}$, up to the action of Aut $\Delta_{2}$, we can suppose that $\gamma$ is given by (1.1). First of all we restate Theorem 2.6 on $\Delta_{2}$ by means of the Cayley transform $\mathcal{C}=\left(\mathcal{C}_{1}, \mathcal{C}_{2}\right): \Delta_{2} \rightarrow H_{2}$.

Corollary 2.8. Let $f: \Delta_{2} \rightarrow \Delta_{2}$ be a holomorphic map which commutes with $\gamma$ given by (1.1). Then there exist $t_{1} \in \mathbf{R}$ and $\hat{g}, \hat{\beta} \in \operatorname{Hol}(X, \mathbf{C})$ such that

a) $f(z)=$

$$
\frac{\left(\cosh t_{1} z_{1}+\sinh t_{1}+i e^{t_{1}} z_{2}^{2} e^{-i \theta_{2} \log \mathcal{C}_{1}(z) / \log \lambda} \hat{\beta}(\pi(\mathcal{C}(z))) / 2\left(1-z_{1}\right), z_{2} \hat{g}(\pi(\mathcal{C}(z)))\right)}{\cosh t_{1}+z_{1} \sinh t_{1}+i e^{t_{1}} z_{2}^{2} e^{-i \theta_{2} \log \mathcal{C}_{1}(z) / \log \lambda} \hat{\beta}(\pi(\mathcal{C}(z))) / 2\left(1-z_{1}\right)}
$$

and

b) $\operatorname{Im}\left(\mathcal{C}_{1}(z)+\mathcal{C}_{2}^{2}(z) e^{-i \theta_{2} \log \mathcal{C}_{1}(z) / \log \lambda} \hat{\beta}(\pi(\mathcal{C}(z)))\right)>\left|\mathcal{C}_{2}(z) \hat{g}(\pi(\mathcal{C}(z)))\right|^{2} \quad \forall z \in \Delta_{2}$.

Vice versa, let $f$ be as in a), where $\hat{g}, \hat{\beta} \in \operatorname{Hol}(X, \mathbf{C})$ satisfy $b)$. Then $f: \Delta_{2} \rightarrow \Delta_{2}$ commutes with $\gamma$.

Now we study the possible fixed points sets of $f$ : in sharp contrast with the one-dimensional case we prove that a map which commutes with a hyperbolic automorphism of $\Delta_{2}$ can have fixed points in $\Delta_{2}$ and we find an explicit form for the possible fixed points sets of $f$, giving also a necessary and sufficient condition for $f$ to have fixed points.

Proposition 2.9. Let $f: \Delta_{2} \rightarrow \Delta_{2}$ be a holomorphic map which commutes with the hyperbolic automorphism $\gamma$ given by (1.1). If $f$ has fixed points in $\Delta_{2}$, then either $f=i d_{\Delta_{2}}$ or $f$ ixf $=\Delta \times\{0\}$. Moreover, $f$ has fixed points in $\Delta_{2}$ iff $f_{1}\left(z_{1}, 0\right)=z_{1}$ for all $z_{1} \in \Delta$.

Proof. We recall that the fixed points set of a holomorphic self-map of $\Delta_{n}$ is given by the intersection of a complex affine space with $\Delta_{n}$; therefore, if $n=2$, fixf is always contained in a complex affine line, unless $f=i d_{\Delta_{2}}$. 
Let $a, b, c \in \mathbf{C}$ be such that the affine line defined by $a z_{1}+b z_{2}+c=0$ contains the fixed points set of $f$, and let $z^{0}=\left(z_{1}^{0}, z_{2}^{0}\right)$ be a fixed point of $f$. Since $\gamma$ and $f$ commute, it is easy to see that $\gamma^{m}\left(z^{0}\right)$ belongs to fixf for all $m \in \mathbf{Z}$. Calling in the form of $\gamma$ given by (1.1) we obtain that

$$
a \frac{\cosh m t_{0} z_{1}^{0}+\sinh m t_{0}}{\sinh m t_{0} z_{1}^{0}+\cosh m t_{0}}+b \frac{e^{i m \theta_{2}} z_{2}^{0}}{\sinh m t_{0} z_{1}^{0}+\cosh m t_{0}}+c=0 \quad \forall m \in \mathbf{Z} .
$$

This is equivalent to

$$
\left(a z_{1}^{0}+c\right) \cosh m t_{0}+\left(c z_{1}^{0}+a\right) \sinh m t_{0}+b e^{i m \theta_{2}} z_{2}^{0}=0 \quad \forall m \in \mathbf{Z} .
$$

Divide both members of the last equation by $\cosh m t_{0}$ and take the limit both for $m \rightarrow+\infty$ and for $m \rightarrow-\infty$. Since $\lim _{m \rightarrow \pm \infty} \cosh m t_{0}=+\infty, \lim _{m \rightarrow \pm \infty} \tanh m t_{0}$ $= \pm 1$ and since the modulus of $b e^{i m \theta_{2}} z_{2}^{0}$ is bounded when $m$ diverges, we obtain

$$
\left(a z_{1}^{0}+c\right)+\left(c z_{1}^{0}+a\right)=0 \quad \text { and } \quad\left(a z_{1}^{0}+c\right)-\left(c z_{1}^{0}+a\right)=0 .
$$

Since $\left|z_{1}^{0}\right|<1$, the last equations imply $a=c=0$. Then the complex affine line containing fixf is the complex line $z_{2}=0$, and hence $z_{2}^{0}=0$. Propositions 1.3 and 1.4 show that, on the complex line $z_{2}=0$, the function $f$ is given by

$$
f\left(z_{1}, 0\right)=\left(\frac{\cosh t_{1} z_{1}+\sinh t_{1}}{\sinh t_{1} z_{1}+\cosh t_{1}}, 0\right) .
$$

Therefore, if $\left(z_{1}^{0}, 0\right)$ is a fixed point for $f$, we obtain that $t_{1}=0$ and hence the set $\Delta \times\{0\}$ is contained in $f i x f$. The assertion follows.

\section{REFERENCES}

1. M. Abate, Iteration theory of holomorphic mappings on taut manifolds, Mediterranean Press, Rende, 1989. MR 92i:32032

2. M. Abate, J.-P. Vigué Common fixed points in hyperbolic Riemann surfaces and convex domains, Proc. Amer. Math. Soc. 112 (1991), 503-512. MR 91i:32027

3. C.C. Cowen, Commuting analytic functions, Trans. Am. Math. Soc. 283 (1984), 685-695. MR 85i:30054

4. C. de Fabritiis, G. Gentili, On holomorphic maps which commute with hyperbolic automorphisms, to appear in Advances in Math., 1995.

5. T. Franzoni, E. Vesentini, Holomorphic maps and invariant distances, North-Holland, Amsterdam, 1980. MR 82a:32032

6. M.H. Heins, A generalization of the Aumann-Carathéodory "Starrheitssatz", Duke Math. J. 8 (1941), 312-316. MR 3:81a

7. S. Krantz, Function theory of several complex variables, Wiley, New York, 1982. MR 84c:32001

8. M. Kuczma, Functional equations in a single variable, PWN, Warzawa, 1968. MR 37:4441

9. M. Kuczma, An introduction to the theory of functional equations and inequalities: Cauchy's equation and Jensen's inequality, PWN, Warzawa, 1985. MR 86i:39008

10. W. Rudin, Function theory in the unit ball of $\mathbf{C}^{n}$, Springer, Berlin, 1980. MR 82i:32002

11. A.L. Shields, On fixed points of commuting analytic functions, Proc. Amer. Math. Soc. 15 (1964), 703-706.

International School for Advanced Studies, via Beirut 2-4, 34014, Trieste, Italy E-mail address: FABRITII@NEUMANN.SISSA.IT 